PROCEEDINGS OF THE

AMERICAN MATHEMATICAL SOCIETY

Volume 140, Number 2, February 2012, Pages 615-620

S 0002-9939(2011)10916-X

Article electronically published on June 13, 2011

\title{
A SHARP REGULARITY RESULT OF SOLUTIONS OF A TRANSMISSION PROBLEM
}

\author{
GIOVANNA CITTI AND FAUSTO FERRARI
}

(Communicated by Matthew J. Gursky)

\begin{abstract}
In this paper we study the regularity of solutions of a transmission problem arising in studying a fiber-reinforced composite media. It is described through a divergence type equation $\operatorname{div}(A \nabla u)=h$, in an open set $D$, where $h$ is a bounded function and $A$ is a uniformly elliptic matrix, bounded and with piecewise Hölder continuous coefficients. The subdomains $D_{i}$ where $A$ is of class $C^{\alpha}$ have disjoint closure and are of class $C^{1, \alpha}$. Exploiting an idea contained in a paper by $\mathrm{Li}$ and Vogelius, we obtain the optimal regularity result for solutions, proving that they are of class $C^{1, \alpha}\left(\bar{D}_{i}\right)$.
\end{abstract}

\section{INTRODUCTION}

In this paper we show that the solutions of a divergence form elliptic equation with piecewise Hölder continuous coefficients have optimal regularity. The problem arises in studying a fiber-reinforced composite media described as a domain $D \subset R^{n}$ with $l-1$ subdomains $D_{m}$ representing the fibers. The domains $D_{m}$ are compactly embedded in $D$, with $C^{1, \alpha}$ boundary and non-intersecting closure. We will also denote $D_{l}=D-\bigcup_{m=1}^{l} D_{m}$. On this set we study the differential equation

$$
\operatorname{div}(A \nabla u)=h+\operatorname{div} g, \text { in } D,
$$

where $A$ is a uniformly elliptic matrix and $A_{\left.\right|_{\bar{D}_{m}}} \in C^{\mu}, g_{\left.\right|_{\bar{D}_{m}}} \in C^{\mu}\left(D_{m}\right)$, and $h \in L^{\infty}(D)$.

In [6] and [11] examples of solutions whose gradient becomes unbounded have been provided, and in [1] the speed of blow up of the gradient has been estimated. We would like to cite also [2] and [3], where very fine estimates have been provided for the degenerate conductivity cases.

On the other hand, if the matrix $A$ is uniformly elliptic, it is well known from the De Giorgi Nash theory (see [7]) that all the solutions belong to $C^{0, \beta}$, where $0<$ $\beta=\min \{\mu, \alpha\}$. Some higher regularity results have been established using the fact that $u$ is a weak solution across the boundary. Indeed, under the assumption that $A$ is diagonal, it has been shown in 5 that the gradient remain bounded even for

Received by the editors August 2, 2010 and, in revised form, November 29, 2010

2010 Mathematics Subject Classification. Primary 35J20; Secondary 35B65.

Key words and phrases. Transmission problems, sharp regularity.

The first and second authors were partially supported by M.U.R.S.T., Italy, and CG-DICE project, EU VII Framework Program.

The second author was partially supported by the GNAMPA project "Equazioni non lineari su varietà: proprietà qualitative e classificazione delle soluzioni".

(C)2011 American Mathematical Society 
circular touching fibers $D_{m}$. Successively Li Vogelius [9] established a general result for solutions of equations (1.1) for general uniformly elliptic matrix $A$ with piecewise Hölder continuous coefficients. Their proof implies in particular that the solution is of class $C_{l o c}^{1, \beta}$, with $\beta=\min \left\{\mu, \frac{\alpha}{(\alpha+1) n}\right\}$. We also refer to the paper [8], where a similar result has been proved for systems. These results allow the sub-domain $D_{m}$ to be tangent, but the exponent of regularity does not seem to be optimal. Recently [10] studied the problem with a completely different technique, based on instruments of complex analysis, and, assuming that the domain $D_{m}$ do not become tangent, together with slightly more restrictive assumption on the structure of the elliptic equation, obtained $C_{l o c}^{1, \beta}$ regularity for the solution of the problem, for all $\beta<\min \{\mu, \alpha\}$. It is then natural to ask if, at least in this non-tangential situation, the optimal regularity $C_{l o c}^{1, \beta}$, with $\beta=\min \{\mu, \alpha\}$, can be achieved. This is exactly the scope of this paper. We prove that in the absence of tangency between the boundaries of the $D_{i}$, slightly modifying the proof of [9] it is possible to obtain the optimal regularity for solutions.

Precisely, we assume that $A$ is a symmetric, positive definite matrix-valued function such that there exist $\bar{\lambda}, \bar{\Lambda}>0$ such that for every $x \in D$ and for every $\xi \in \mathbb{R}^{n} \backslash\{0\}$,

$$
\bar{\lambda}|\xi|^{2} \leq \sum_{i, j=1}^{n} a_{i j}(x) \xi_{i} \xi_{j} \leq \bar{\Lambda}|\xi|^{2} .
$$

Moreover, we set, following the notation in [9]:

$$
\begin{gathered}
A(x)=A^{(m)}(x), x \in D_{m}, 1 \leq m \leq l . \text { Then } A^{(m)}(x) \in C^{\mu}\left(\bar{D}_{m}\right) . \\
g^{(m)} \in C^{\mu}\left(\bar{D}_{m}, \mathbb{R}^{n}\right), \quad g(x)=g^{(m)}(x), x \in D_{m}, 1 \leq m \leq l, \\
h \in L^{\infty}(D), \\
\phi \in C^{1, \mu}(\partial D) .
\end{gathered}
$$

We can now state our main result.

Theorem 1.1. Let $A, g$ and $h$ satisfy (1.3)-(1.6). Suppose $\varepsilon>0$. There exists a constant $C=C\left(D, n, \alpha^{\prime}, \alpha, \varepsilon, \bar{\lambda}, \bar{\Lambda},\left\|A^{(m)}\right\|_{C^{\alpha^{\prime}}\left(\bar{D}_{m}\right)}\right)$ and the $C^{1, \alpha}$ modulus of $\bigcup_{m=1}^{l} \partial D_{m}$ such that if $u \in H^{1}(D)$ is a solution of (1.1) with boundary datum $u=\phi$, then

$$
\max _{1 \leq m \leq M}\|u\|_{C^{1, \beta}\left(\bar{D}_{m} \cap D_{\varepsilon}\right)} \leq C\left(\|u\|_{L^{\infty}(D)}+\|h\|_{L^{\infty}(D)}+\max _{1 \leq m \leq l}\left\|g^{(m)}\left(\bar{D}_{m}\right)\right\|\right),
$$

where $\beta=\min (\alpha, \mu)$ and $D_{\varepsilon}=\{x \in D: \operatorname{dist}(x, \partial D)>\varepsilon\}$.

\section{Proof of the MAIN THEOREM}

We first note that since the result is local and the boundary of the set $D_{m}$ is disjoint, we can fix a point $x \in \partial D_{m}$ and we can locally perform a change of coordinates $\psi$ of class $C^{1, \alpha}$ in such a way that $\psi\left(\partial D_{m}\right)$ in a neighborhood of the fixed point is a plane. Precisely, we can find a neighborhood $U$ of $x$ such that $U \cap D_{j} \neq \emptyset$ if and only if $j=m$ or $j=l, \psi(U)$ is an $n$-dimensional cube $\Omega$ of size 2 parallel to the axis, and the images of the regions $D_{m} \cap U$ and $D_{l} \cap U$ become respectively

$$
\Omega^{+}=\left\{x \in \Omega, 0<x_{n}\right\}, \quad \Omega^{-}=\left\{x \in \Omega, \quad x_{n}<0\right\} .
$$


We represent the set $\Omega$ as the union of slices

$$
\Omega_{m}=\left\{x \in \Omega, \quad c_{m-1}<x_{n}<c_{m}\right\}, 1 \leq m \leq l+1,
$$

choosing one of the points $c_{m}=0$ so that one of the boundaries of $\Omega_{m}$ is the image of the boundary of $D_{m} \cap U$.

The proof is a modification of the one contained in [9]. Hence we have to locally approximate the problem with a new one, with piecewise constant coefficients.

With $\overline{\mathcal{A}}(\bar{\lambda}, \bar{\Lambda})$ we denote the set of symmetric matrices constant on the regions $\Omega_{j}, j=1, \ldots, l+1$, and with smallest and largest eigenvalue, respectively $\bar{\lambda}, \bar{\Lambda}$. For every matrix $\bar{A} \in \overline{\mathcal{A}}(\bar{\lambda}, \bar{\Lambda})$ we will call

$$
\bar{A}_{i, j}(x)=\bar{A}_{i j}^{m}
$$

the constant value attained on $\Omega_{m}, 1 \leq m \leq l+1$. Analogously we will indicate $\bar{G}$ vectors constant on each considered slice $\Omega_{m}$ :

$$
\bar{G}(x)=G^{(m)}, x \in \Omega_{m}, 1 \leq m \leq l+1 .
$$

Definition 2.1. For any $s>0$ and any $p \in(1, \infty)$ we define the norm

$$
\|h\|_{Y^{s, p}}:=\sup _{0<r \leq 1} r^{1-s}\left(\int_{r \Omega}|h|^{p}\right)^{1 / p} .
$$

With this notation we recall the following result proved in Proposition 3.2 in 9 . Below we give a version of such result useful for the subsequent proofs of our paper:

Proposition 2.2. Suppose $g=\left(g_{1}, \ldots, g_{n}\right) \in L^{q}(\Omega), h \in L^{q / 2}(\Omega)$ for some $q>n$. Let $\bar{\alpha} \in(0,1)$ and let $u \in H^{1}(\Omega)$ be a solution to

$$
\partial_{i}\left(A_{i j}(x) \partial_{j} u\right)=h+\partial_{i} g_{i}, \text { in } \Omega,
$$

with

$$
\|u\|_{L^{\infty}(\Omega)} \leq 1 .
$$

There exist constants $\sigma \in\left(0, \frac{1}{4}\right), \epsilon_{0}>0$, and $C>0, C=C(n, q, \bar{\alpha}, \bar{\lambda}, \bar{\Lambda})$ such that if

$$
\begin{gathered}
\|A-\bar{A}\|_{Y^{1+\bar{\alpha}, q}} \leq \epsilon_{0}, \quad\|g-\bar{G}\|_{Y^{1+\bar{\alpha}, q}}+\|h-\bar{H}\|_{Y^{1+\bar{\alpha}, q / 2}} \leq \epsilon_{0}, \\
\|\bar{G}\|_{L^{\infty}(\Omega)}+\|\bar{H}\|_{L^{\infty}(\Omega)} \leq 1,
\end{gathered}
$$

then we can find a sequence of continuous, piecewise linear functions $p_{k}$,

$$
p_{k}(x)=a_{k}^{(m)}+b_{k}^{(m)} x, x \in \Omega_{m} \cap\left[-\frac{1}{4}, \frac{1}{4}\right]^{n},
$$

such that

$$
\left\|u-p_{k}\right\|_{L^{\infty}\left(\sigma^{k} \Omega\right)} \leq \sigma^{k(1+\bar{\alpha})} .
$$

The limit $p(x)=\lim _{k \rightarrow \infty} p_{k}(x)$ exists for $x \in \frac{1}{4} \Omega$ is a continuous piecewise linear function with coefficients that are uniformly bounded by $C$ and furthermore satisfies

$$
\partial_{i}\left(\bar{A}_{i j}(x) \partial_{j} p\right)=\partial_{i}\left(\bar{G}_{i}\right) \text {, in } \frac{1}{4} \Omega,
$$

and

$$
|u(x)-p(x)| \leq C|x|^{1+\bar{\alpha}} \text {, in } \frac{1}{4} \Omega .
$$

Let us now prove the main approximation properties of the matrix $A$ on the set $\Omega$ and on a rescaled version of it: 
Lemma 2.3. Let $B$ be the ball of radius $r$. Assume that $A \in C^{0, \mu}\left(B^{+}\right)$and $A \in$ $C^{0, \mu}\left(B^{-}\right)$. Let us define $\bar{A}$ as $A^{+}(0)$ if $x_{n}>0$ and as $A^{-}(0)$ if $x_{n}<0$. There exists a positive constant $C$ such that

$$
\left(f_{B}|A-\bar{A}|^{q} d x\right)^{1 / q} \leq C r^{\mu} .
$$

Proof. Then

$$
\left(f_{B}|A-\bar{A}|^{q} d x\right)^{1 / q}=\frac{1}{|B|}\left(\int_{B^{+}}|A-\bar{A}|^{q} d x+\int_{B^{-}}|A-\bar{A}|^{q} d x\right)^{1 / q} .
$$

Thus

$$
\begin{aligned}
\left(f_{B}|A-\bar{A}|^{q} d x\right)^{1 / q} & \leq \frac{1}{|B|}\left(L_{1} \int_{B^{+}}|x|^{\mu q} d x+L_{2} \int_{B^{-}}|x|^{\mu q} d x\right)^{1 / q} \\
& \leq \max \left\{L_{1}, L_{2}\right\} \frac{1}{|B|}\left(\int_{B}|x|^{\mu q} d x\right)^{1 / q} \\
& =\max \left\{L_{1}, L_{2}\right\} \frac{\omega_{n-1}}{|B|}\left(\int_{0}^{r} \rho^{\mu q+n-1} d x\right)^{1 / q} \\
& =C\left(r^{\mu q+n-n}\right)^{1 / q}=C r^{\mu} .
\end{aligned}
$$

We define $A_{r_{0}}=A\left(r_{0} x\right), \bar{A}_{r_{0}}(x)=\bar{A}\left(r_{0} x\right)$.

Lemma 2.4. Suppose that $q>n$ and $0<\alpha^{\prime} \leq \min \{\mu, \alpha\}$. Given any $\epsilon_{0}>0$, depending on $n, q, \quad l, \epsilon_{0}, \alpha, \quad \alpha^{\prime}, \bar{\lambda}, \bar{\Lambda}, \max _{1 \leq m \leq l}\left\|f_{m}\right\|_{C^{1, \alpha}[-1,1]^{n-1}}$, and $\max _{1 \leq m \leq l}\left\|A^{(m)}\right\|_{C^{\alpha^{\prime}\left(\tilde{D}_{m}\right)}}$, we have that

$$
\left(f_{r \Omega}\left|A_{r_{0}}(x)-\bar{A}_{r_{0}}(x)\right|^{q}\right)^{1 / q} \leq \epsilon_{0} r^{\mu} \leq \epsilon_{0} r^{\alpha^{\prime}},
$$

for every $r, 0<r \leq 1$.

Now combining Proposition 2.2 with Lemma 2.4 we can prove the following.

Proposition 2.5. Let $A \in \mathcal{A}(\bar{\lambda}, \bar{\Lambda})$ and $\bar{A} \in \overline{\mathcal{A}}(\bar{\lambda}, \bar{\Lambda})$. Assume that $h \in L^{\infty}(\Omega)$. For any $q>n$, and any $0<\alpha^{\prime} \leq \min \{\mu, \alpha\}$ ther $\mathbb{1}^{1}$ exist constants $C$ and $r_{0}$ such that if $u \in H^{1}(\Omega)$ is a solution to

$$
\partial_{i}\left(A_{i j}(x) \partial_{j} u\right)=h+\partial_{i} g_{i}, \text { in } \Omega,
$$

with

$$
\|u\|_{L^{\infty}(\Omega)}+\|h\|_{L^{\infty}(\Omega)}+\max _{1 \leq m \leq l+1}\left\|g^{(m)}\right\|_{C^{\alpha^{\prime}} \overline{\tilde{D}}_{m}} \leq 1,
$$

then one may find a continuous, piecewise linear function, $p$, whose coefficients are bounded in absolute value by $C$, and which satisfies

and 2

$$
\partial_{i}\left(A_{i j}(x) \partial_{j} p\right)=\partial_{i} G_{i}, \text { in } r_{0} \Omega,
$$

$$
|u(x)-p(x)| \leq C|x|^{1+\alpha^{\prime}}, x \in r_{0} \Omega .
$$

The constant $C$ and $r_{0}$ depend on $n, \alpha^{\prime}, \alpha, q, \bar{\lambda}, \bar{\Lambda}$, the number $l$, $\max _{1 \leq m \leq l+1}\left\|A^{(m)}\right\|_{C^{\alpha^{\prime}} \overline{\tilde{D}}_{m}}$, and $\max _{1 \leq m \leq l+1}\left\|f^{(m)}\right\|_{C^{1, \alpha}[-1,1]^{n-1}}$.

\footnotetext{
${ }^{1}$ Here $\alpha$ appears. We get the best exponent because the boundary is flat. Indeed, in the paper by $\mathrm{Li}$ and Vogelius (see [9]) there is $\frac{\alpha}{(\alpha+1) q}$.

${ }^{2}$ Notice that in the following inequality the best exponent is $1+\min \{\mu, \alpha\}$, while in $[9$ it is $1+\min \left\{\mu, \frac{\alpha}{(\alpha+1) q}\right\}$.
} 
Proof. The proof is similar to Proposition 5.3 in [9]. For the reader's convenience we present the proof of this assertion. We consider $w(x)=u\left(r_{0} x\right)$, which solves the equation

$$
\partial_{i}\left(\left(A_{r_{0}}\right)_{i j} \partial_{j} w\right)=r_{0}^{2} h+r_{0} \partial_{i} g_{i}, \text { in } \Omega .
$$

Then we choose $r_{0}$ as prescribed in Lemma 2.4 and obtain the estimate

$$
\left\|A_{r_{0}}-\bar{A}_{r_{0}}\right\|_{Y^{1+\alpha^{\prime}, q}} \leq \epsilon_{0}
$$

which allows us to apply Proposition 2.2. Applying Lemma 2.4 to the functions $g$ and $\bar{G}$ we may select $r_{0}$ sufficiently small that

$$
\left\|g\left(r_{0} \cdot\right)-G\left(r_{0} \cdot\right)\right\|_{Y^{1+\alpha^{\prime}, q}} \leq \frac{1}{\epsilon_{0}} .
$$

By selecting $r_{0}$ sufficiently small we thus get

$$
r_{0}^{2}\left\|h\left(r_{0} \cdot\right)\right\|_{Y^{1+\alpha^{\prime}, q / 2}}+r_{0}\left\|g\left(r_{0} \cdot\right)-\bar{G}\left(r_{0} \cdot\right)\right\|_{Y^{1+\alpha^{\prime}, q}} \leq r_{0}^{2}\|h\|_{L^{\infty}}+r_{0} \frac{\epsilon_{0}}{2} \leq \epsilon_{0} .
$$

We also have

$$
r_{0}\left\|G\left(r_{0} \cdot\right)\right\|_{L^{\infty}(\Omega)} \leq r_{0} \max _{1 \leq m \leq l}\left\|g^{(m)}\right\|_{C^{\alpha^{\prime}\left(\bar{D}_{m}\right)}} \leq 1 .
$$

We can now apply Proposition 2.2 to $u$. This leads to the existence of a continuous, piecewise linear polynomial $q$ whose coefficients are bounded in absolute value by $C$ and which satisfies

$$
\partial_{i}\left(\left(\bar{A}_{r_{0}}\right)_{i j} \partial_{j} q\right)=r_{0}^{2} h+r_{0} \partial_{i} \bar{G}_{i}, \text { in } \Omega .
$$

The function $p(x)=q\left(x / r_{0}\right)$ satisfies all the requirements from the statement of this proposition.

The statement of Proposition 2.5 is analogous to Proposition 5.3 in Li and Vogelius [9], but, under the non-tangency assumption we made here on the boundary, we obtain a Hölder estimate of exponent $\alpha^{\prime}$ instead of the exponent $\alpha^{\prime}=\frac{\alpha}{(\alpha+1) q}$ obtained in 9 . Hence at this point the proof of the main theorem, Theorem 1.1. is the same as for Theorem 1.1 in [9] on p. 122 .

\section{ACKNOWLEDGMENT}

The authors are grateful to Professor J. Verdera for pointing out the problem and for useful conversations on the subject of the paper.

\section{REFERENCES}

1. H. Ammari, H. Kang, and M. Lim, Gradient estimates for solutions to the conductivity problem, Math. Ann. 332 (2005), 277-286. MR2178063 (2006h:78010)

2. H. Ammari, H. Kang, H. Lee, J. Lee, and M. Lim, Optimal estimates for the electric field in two-dimensions, Journal de Mathématiques Pures et Appliquées 88 (2007), 307-324. MR2384571 (2009a:35043)

3. H. Ammari, H. Kang, H. Lee, M. Lim, and H. Zribi, Decomposition theorems and fine estimates for electrical fields in the presence of closely located circular inclusions, Journal of Differential Equations 247 (2009), 2897-2912. MR2569851 (2010j:35102)

4. I. Babuska, B. Andersson, P.J. Smith, and K. Levin, Damage analysis of fiber composites. Part I: Statistical analysis on fiber scale, The Aeronautical Research Institute of Sweden, Technical Note 15 (1998).

5. E. Bonnetier and M. Vogelius, An elliptic regularity result for a composite medium with touching fibers of circular cross-section, SIAM J. Math. Anal. 31 (2000), 651-677. MR.1745481 (2002a:35052) 
6. B. Budiansky and G.F. Carrier, High shear stresses in stiff fiber composites. J. App. Mech. 51 (1984), 733-735.

7. D. Gilberg and N. S. Trudinger, Elliptic Partial Differential Equations of Second Order, Springer-Verlag, 1983. MR737190 (86c:35035)

8. Y.Y. Li and L. Nirenberg, Estimates for elliptic systems from composite material, Commun. Pure Appl. Math. 56 (2003), 892-925. MR1990481 (2004k:35097)

9. Y.Y. Li and M. Vogelius, Gradient estimates for solutions to divergence form elliptic equations with discontinuous coefficients, Arch. Ration. Mech. Anal. 153 (2000), 91-151. MR.1770682 (2001m:35083)

10. J. Mateu, J. Orobitg, and J. Verdera, Extra cancellation of even Caldern-Zygmund operators and quasiconformal mappings, J. Math. Pures Appl. 91 (2009), 402-431. MR2518005 (2010f:42026)

11. X. Markenscoff, Stress amplification in vanishingly small geometries, Computational Mechanics 19 (1996), 77-83.

12. M. Schechter, A generalization of the problem of transmission, Ann. Scuola Norm. Sup. Pisa 14 (1960), 207-236. MR0131063 (24:A917)

Dipartimento di Matematica, Università Degli Studi di Bologna, Piazza di Porta S. Donato, 5, 40126 Bologna, Italy

E-mail address: citti@dm.unibo.it

Dipartimento di Matematica, Università Degli Studi di Bologna, Piazza di Porta S. Donato, 5, 40126 Bologna, Italy

E-mail address: ferrari@dm.unibo.it 